PROCEEDINGS OF THE

AMERICAN MATHEMATICAL SOCIETY

Volume 135, Number 1, January 2007, Pages 47-49

S 0002-9939(06)08457-7

Article electronically published on June 30, 2006

\title{
A FEW UNCAUGHT UNIVERSAL HERMITIAN FORMS
}

\author{
JAE-HEON KIM AND POO-SUNG PARK
}

(Communicated by Wen-Ching Winnie Li)

\begin{abstract}
We will complete the list of universal binary Hermitian forms over imaginary quadratic fields by investigating three Hermitian forms missed by previous researchers.
\end{abstract}

\section{INTRODUCTION}

The celebrated Four Square Theorem by Lagrange 4 states that any positive integer is a sum of four squares. This has been generalized by many mathematicians in many directions. In particular, Ramanujan [7] found all 54 positive definite diagonal quaternary quadratic forms representing all positive integers. We call a quadratic form universal if it represents all positive integers. Recently, several people studied the problem analogous to universal forms over the imaginary quadratic fields. Earnest and Khosravani [1] defined a universal Hermitian form as a positive definite one representing all positive integers, and they found 13 universal binary Hermitian forms over the imaginary quadratic fields of class number 1. More generally, Iwabuchi 2] investigated Hermitian lattices and found 9 lattices over the imaginary quadratic fields of class number bigger than 1.

We have discovered that they missed a few universal binary Hermitian forms. These are $x \bar{x}+3 y \bar{y}$ in $\mathbb{Q}(\sqrt{-7})$ and $x \bar{x}+4 y \bar{y}$ and $x \bar{x}+5 y \bar{y}$ in $\mathbb{Q}(\sqrt{-2})$. These complete the Earnest-Khosravani-Iwabuchi list of binary universal Hermitian lattices over imaginary quadratic number fields.

\section{Preliminaries}

Let $E$ be an imaginary quadratic field over $\mathbb{Q}$ and let $m>0$ be a square-free integer for which $E=\mathbb{Q}(\sqrt{-m})$. We denote the $\mathbb{Q}$-involution by ${ }^{-}$and the ring of integers in $E$ by $\mathcal{O}$.

Let $V$ be an $n$-dimensional Hermitian space over $E$ with nondegenerate Hermitian form $H$. A finitely generated $\mathcal{O}$-module $L$ in $V$ is called a Hermitian lattice.

In the case that $E$ is a field of class number $1, \mathcal{O}$ is a principal ideal domain and thus every Hermitian lattice is free. Then for a suitable basis $\left\{v_{1}, \cdots, v_{n}\right\}$ of $L$ we can think of a Hermitian form as a function $f: \mathcal{O}^{n} \rightarrow \mathbb{Z}$ defined by $f\left(x_{1}, \cdots, x_{n}\right)=H\left(\sum x_{i} v_{i}\right)=\sum H\left(v_{i}, v_{j}\right) x_{i} \overline{x_{j}}$.

Received by the editors March 25, 2005 and, in revised form, July 5, 2005 and August 9, 2005. 2000 Mathematics Subject Classification. Primary 11E39; Secondary 11E20, 11E41.

Key words and phrases. Universal Hermitian form.

The second author was partially supported by KRF(2003-070-c00001).

(C)2006 American Mathematical Society Reverts to public domain 28 years from publication 
We can regard $(V, H)$ as a $2 n$-dimensional quadratic space $(\tilde{V}, B)$ over $\mathbb{Q}$ as defined in [3], where $B(x, y)=\frac{1}{2} \operatorname{Tr}_{E / \mathbb{Q}}(H(x, y))$. Similarly we can associate a quadratic lattice $\tilde{L}$ with the Hermitian lattice $L$. The ring $\mathcal{O}$ of integers in $E$ has a basis $\{1, \omega\}$ as a $\mathbb{Z}$-module of rank 2 , where $\omega=\frac{1+\sqrt{-m}}{2}$ if $m \equiv 3(\bmod 4)$ and $\omega=\sqrt{-m}$ otherwise. Then $\tilde{f}\left(x_{1}, y_{1}, \cdots, x_{n}, y_{n}\right)=f\left(x_{1}+\omega y_{1}, \cdots, x_{n}+\omega y_{n}\right)$ is a quadratic form in $2 n$ variables corresponding to the lattice $\tilde{L}$.

\section{MAIN RESUlt}

From now on we will consider only binary Hermitian forms and their corresponding quaternary quadratic forms. The binary Hermitian form $f$ can be written as $f(x, y)=a x \bar{x}+b x \bar{y}+\bar{b} \bar{x} y+c y \bar{y}$ with $a, c \in \mathbb{N}, b=b_{1}+\omega b_{2}, b_{1}, b_{2} \in \mathbb{Z}$. We can reduce any binary Hermitian form to one whose coefficients satisfy:

$$
a=\min f,-\frac{1}{2} a \leq b_{1} \leq \frac{1}{2} a, 0 \leq b_{2} \leq \frac{1}{2} a, \text { and } a \leq c .
$$

Since any universal Hermitian form must represent 1, a must equal 1 and $b$ must vanish for such a form. Thus we may consider only diagonal Hermitian forms $x \bar{x}+c y \bar{y}$.

In [1, the screening process was performed by using representation of the integers 1 through 5. But exactly three forms were missed and all of them are universal.

Theorem. The binary Hermitian forms $x \bar{x}+3 y \bar{y}$ in $\mathbb{Q}(\sqrt{-7})$ and $x \bar{x}+4 y \bar{y}$ and $x \bar{x}+5 y \bar{y}$ in $\mathbb{Q}(\sqrt{-2})$ are universal.

Proof. The associated quaternary quadratic forms of $x \bar{x}+4 y \bar{y}$ and $x \bar{x}+5 y \bar{y}$ over $\mathbb{Q}(\sqrt{-2})$ are $\langle 1,2,4,8\rangle$ and $\langle 1,2,5,10\rangle$, respectively. Their universality has been shown by Ramanujan $[7$.

The genus of $f=x \bar{x}+3 y \bar{y}$ over $\mathbb{Q}(\sqrt{-7})$ consists of the class of $f$ and the class of $g=2 x \bar{x}+x \bar{y}+\bar{x} y+2 y \bar{y}$. Using the trace map, we get the quaternary quadratic forms corresponding to $f$ and $g$, respectively:

$$
\begin{aligned}
& \tilde{f}=x^{2}+2 y^{2}+3 z^{2}+6 w^{2}+x y+3 z w \\
& \tilde{g}=2 x^{2}+2 y^{2}+4 z^{2}+4 w^{2}+2 x y+2 x z+x w+y z+2 y w+4 z w .
\end{aligned}
$$

We show that the genus of $\tilde{f}$ and $\tilde{g}$ is universal before the universality of $f$. The discriminant is $441=3^{2} \times 7^{2}$ in the sense of Nipp's table [5. Thus if $p \neq 2,3,7$, the genus is locally universal by [6, 92:1b]. If $p=3$, then the Jordan splitting of $\tilde{f}_{p}$ and $\tilde{g}_{p}$ is $\langle 1,1\rangle \perp 3\langle 1,1\rangle$. The genus represents all 3 -adic integers since $\langle 1,1\rangle$ represents all units by [6, 92:1b]. If $p=7$, then the Jordan splitting is $\langle 1, \Delta\rangle \perp 7\langle 1, \Delta\rangle$. Thus the genus represents all 7 -adic integers. When $p=2, \tilde{f}_{p} \cong \tilde{g}_{p} \cong x y+z w$. Hence the genus is locally universal for $p=2$. Finally $\tilde{f}$ and $\tilde{g}$ are positive definite and so the genus is locally universal for any prime $p$.

Now suppose that $n$ is a positive integer represented by $\tilde{g}$. There exist $x, y, z, w \in$ $\mathbb{Z}$ such that

If $x$ is even,

$$
\tilde{g}(x, y, z, w)=n \text {. }
$$

$$
\tilde{f}\left(z+2 w, \frac{x}{2}+y, z, \frac{x}{2}\right)=\tilde{g}(x, y, z, w)=n .
$$

If $x$ is odd and $y$ is even,

$$
\tilde{f}\left(2 z+w, x+\frac{y}{2}, w, \frac{y}{2}\right)=\tilde{g}(x, y, z, w)=n .
$$


If $x$ is odd and $y$ is also odd,

$$
\tilde{f}\left(z-w, \frac{x-y}{2}, z+w, \frac{x+y}{2}\right)=\tilde{g}(x, y, z, w)=n .
$$

Hence $f$ is universal.

\section{REFERENCES}

[1] A. G. Earnest and A. Khosravani, Universal binary Hermitian forms, Math. Comp. 66 (1997), pp.1161-1168. MR.1422787 (98a:11048)

[2] H. Iwabuchi, Universal binary positive definite Hermitian lattices, Rocky Mountain J. Math. 30 (2000), no. 3, pp.951-959. MR.1797825 (2002c:11043)

[3] N. Jacobson, A note on hermitian forms, Bull. Amer. Math. Soc. 46 (1940), pp.264-268. MR0001957 (1:325d)

[4] J. L. Lagrange, Démonstration d'un théorème d'arithmétique, Euvres 3 (1770), pp.189-201.

[5] G. L. Nipp, Quaternary quadratic forms: computer generated tables, Spinger-Verlag, New York, 1991. MR1118842 (92h:11030)

[6] O. T. O'Meara, Introduction to quadratic forms, Spinger-Verlag, New York, 1973. MR.1754311 (2000m:11032)

[7] S. Ramanujan, On the expression of a number in the form $a x^{2}+b y^{2}+c z^{2}+d w^{2}$, Proc. Cambridge Phil. Soc. 19 (1917), pp.11-21.

National Security Research Institute, 161 Gajeong-dong Yuseong-gu, Daejeon, 305350, KOREA

E-mail address: jaeheon@etri.re.kr

Department of Mathematical Sciences, Seoul National University, San 56-1 ShillimDONG GWanaK-Gu, SeOul, 151-747, Korea

E-mail address: sung@math.snu.ac.kr 